\title{
An Atlas of the infrared spectral region
}

\section{The late-type stars $(G-M)^{\star}$}

\author{
J.M. Carquillat ${ }^{1}$, C. Jaschek ${ }^{2}$, M. Jaschek ${ }^{2}$, and N. Ginestet ${ }^{1}$ \\ 1 Observatoire Midi-Pyrénées, Laboratoire d'Astrophysique de Toulouse, UMR. 5572 du CNRS, 14 Avenue Edouard Belin, \\ 31400 Toulouse, France \\ 2 Observatoire Astronomique, URA 1280 du CNRS, 11 Rue de l'Université, 67000 Strasbourg, France
}

Received June 10; accepted August 28, 1996

\begin{abstract}
This Atlas illustrates the behavior of late type stars (F, G, K and M) in the near infrared $8400-8800 \AA$ region with a resolution of about $2 \AA$. Seventeen figures illustrate the spectral sequence and luminosity classes $\mathrm{V}$, III, Ib and Ia. Four figures illustrate peculiar spectra, namely those of Am stars, composites, weak metal stars and $\mathrm{S}$ and $\mathrm{C}$ type objects. The complete Atlas is also available as FITS files from the CDS de Strasbourg and other data centers.
\end{abstract}

Key words: atlases — stars: fundamental parameters stars late type - infrared: stars

\section{Introduction}

In a previous paper (Ginestet et al. 1994) we have shown the interest of the near infrared region for the accurate classification of the cooler components of composite spectra. This is due to the fact that the magnitude difference favors the late-type giant or supergiant companions. In that paper we examined in detail the behavior of the MK standards and we found that with the exception of a few $(6 \%)$, the MK standards can also be used as standards in the near infrared domain $(8380-8780 \AA)$. We provided a number of classification criteria using the equivalent widths $\left(W_{\lambda}\right)$ of a number of features (due for instance to FeI, TiI, CaII, blend at 8468, etc ...) plotted against the MK spectral type and luminosity class of the star. We remarked however that it is not enough to apply simply the criteria, but that the whole spectrum has to be examined carefully. This is entirely in line with similar remarks by the authors of the MK system. We thought therefore that it would be worthwhile to reproduce the spectra themselves, which lead quite naturally to the present Atlas.

\footnotetext{
* based upon observations carried out at the Observatoire de Haute-Provence (CNRS).
}

The present Atlas (Part II) covers the later type stars and is to be taken as a continuation of Part I, which presented the early type stars (Andrillat et al. 1995). Both parts are based on spectra obtained with the same instrument and the same dispersion $(33 \AA / \mathrm{mm})$ covering the same spectral interval. It should be added that a few peculiar stars, signaled in the text, have been obtained with the AURELIE spectrometer. We would like to recall that the near infrared has been studied by a number of authors which we would like to mention briefly.

The infrared region has been studied:

- to emphasize the interest it presents for the obtention of luminosity criteria (Merrill 1934; Keenan \& Hynek 1945) - for the classification of later type stars, essentially those of type M (Sharpless 1956; Parsons 1964; Solf 1978; Barbieri et al. 1981; Kirkpatrick et al. 1991; Huang et al. 1994).

Very often the infrared CaII triplet lines alone have been analysed for a classification of the late type stellar components of galaxies (Jones et al. 1984; Carter et al. 1986; Alloin \& Bicca 1989; Diaz et al. 1989; Zhou 1991; Mallik 1994).

Two atlases should also be signaled, based upon material obtained on a much lower scale. The first one is by Danks \& Dennefeld (1994) and is based upon southern MK standards observed over an extended region $(5800-10200 \AA)$ at a dispersion of $171 \AA / \mathrm{mm}$ and the other one is by Torres-Dodgen \& Weaver (1993). The latter covers the region $5800-8900 \AA$ at approximately $15 \AA$ resolution. In what follows we shall describe first the material used and the reduction procedure (for more details see Ginestet et al. 1994) and then the atlas itself and the classification procedure.

\section{Observations and reduction procedure}

Essentially all spectra reproduced in this Atlas were obtained with the CARELEC spectrograph (Lemaitre et al. 
Table 1. MK standard stars

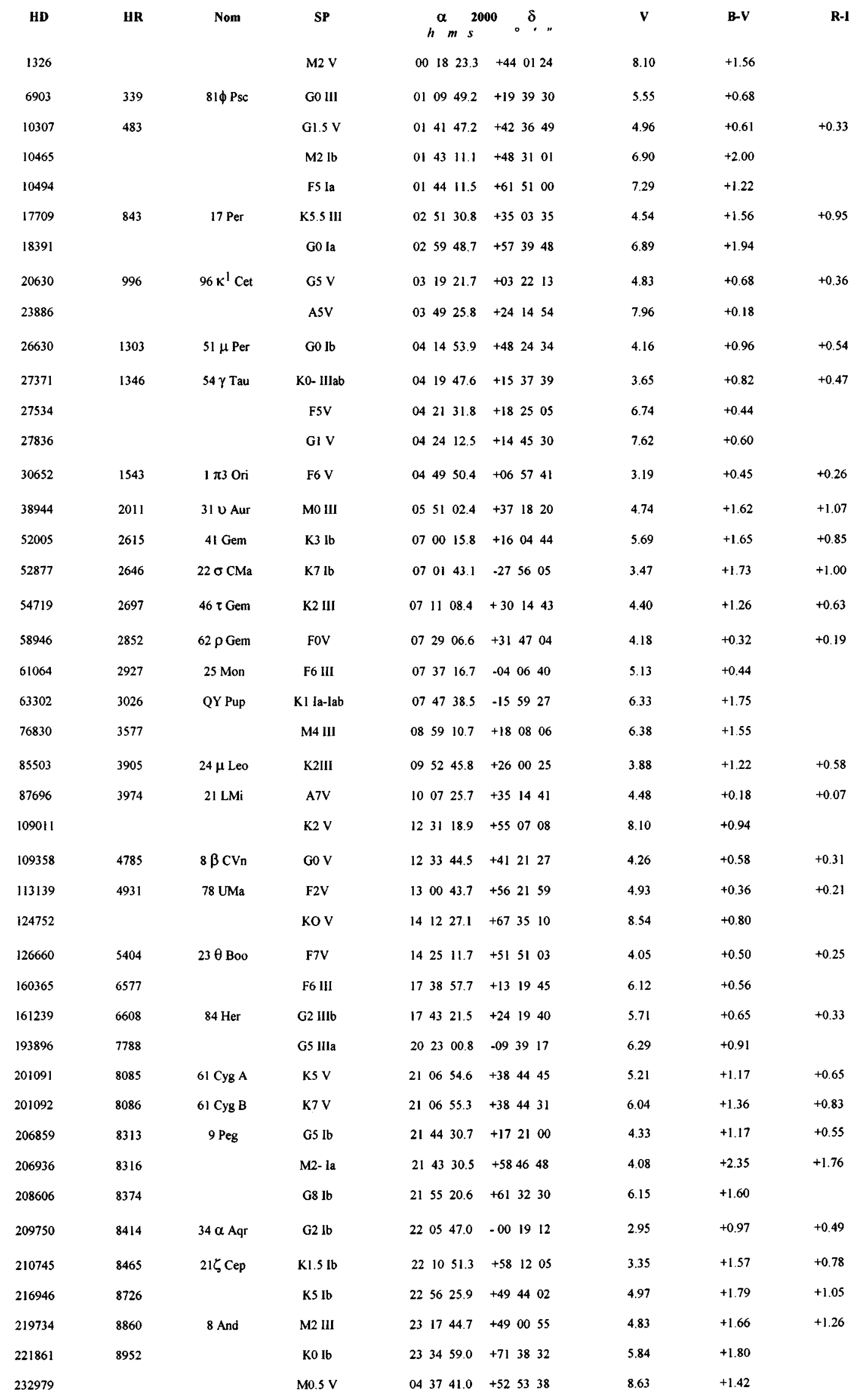


Table 2. Stars with spectral peculiarities

\begin{tabular}{|c|c|c|c|c|c|c|c|c|c|c|}
\hline HD/BD & HR & Name & SP & $h \begin{array}{c}\alpha \\
h\end{array}$ & $\begin{array}{ll}x \\
n\end{array}$ & 000 . & $\mathrm{v}$ & B-V & R-I & figures \\
\hline 6582 & 321 & $30 \mu$ Cas & G5Vp & 0108 & 16.3 & $+54 \quad 55 \quad 14$ & 5.17 & +0.69 & +0.42 & 20 \\
\hline 19445 & & & sd G5 & $03 \quad 08$ & 26.3 & +262035 & 8.05 & +0.46 & & 20 \\
\hline 27749 & 1376 & 63 Tau & $\mathrm{A} 1 \mathrm{~m}$ & 0423 & 25.0 & +164638 & 5.64 & +0.30 & +0.16 & 18 \\
\hline 59604 & & & $\mathrm{G} 21 \mathrm{II}+\mathrm{A} 2$ & 0730 & 56.6 & $\begin{array}{l}+083308 \\
\end{array}$ & 7.20 & & & 19 \\
\hline 83632 & & & K2III & 0940 & 34.2 & $+2600 \quad 17$ & 8.01 & +1.39 & & 20 \\
\hline 92839 & 4195 & VY UMa & C-N5 $\mathrm{C}_{2} 4.5$ & 1045 & 03.9 & +672441 & 6.00 & +2.39 & +1.27 & 21 \\
\hline 93903 & 4237 & 41 Sex & $\mathrm{Am}$ & 1050 & 18.0 & $\begin{array}{lll}-08 & 53 & 52\end{array}$ & 5.79 & +0.16 & +0.07 & 18 \\
\hline 112127 & & & $\mathrm{C}-\mathrm{R} 3 \mathrm{III} \mathrm{C}_{2} 1.5$ & 1253 & 55.6 & +264648 & 6.91 & +1.26 & & $2 l$ \\
\hline$+44^{\circ} 2267$ & & & $\mathrm{~S} 3 \mathrm{Zr} 3 \mathrm{Ti} 1.5$ & 1321 & 18.0 & +435700 & 9.74 & +1.90 & & 21 \\
\hline 184759 & 7441 & 9 Cyg & $\mathrm{G} 8 \mathrm{III}+\mathrm{A} 2 \mathrm{~V}$ & 1934 & 50.8 & +292747 & 5.38 & +0.55 & & 19 \\
\hline 187796 & 7564 & $x$ Cyg & $\mathrm{S} 6+\mathrm{Zr} 2 \mathrm{Ti} 6.5 \mathrm{e}$ & 1950 & 33.8 & +325451 & 4.23 & +1.82 & +2.68 & 21 \\
\hline
\end{tabular}

1990) mounted on the $193 \mathrm{~cm}$ telescope of the OHP observatory. Nine spectra (Figs. 20 and 21) were obtained with the AURELIE spectrograph (Gillet et al. 1994) mounted on the $152 \mathrm{~cm}$ telescope of the OHP. The dispersion of $33 \AA / \mathrm{mm}$ is the same in both cases and leads, with the receivers used (CCD Thomson or Tektronix), to a resolution of the order of $2 \AA$.

The reduction of the observations was made at the OHP with the IHAP program. The images are treated first to eliminate the observational noise (subtraction of an off-set field) and to eliminate the defects of the receiver (division of the spectrum by a flat field given by a tungsten lamp). The spectra were then calibrated in wavelength, using a Ne lamp as calibration source. Next the spectra are straightened and normalised to the continuun, and finally plotted with an HP tracer. Each spectrum is thus an intensity tracing as a function of wavelength, the intensity of the continuun always being set equal to unity.

\section{Content of the Atlas}

The Atlas presents 36 MK standards distributed on 17 sheets (Figs. 1 to 17 ) illustrating the spectral type sequence and the luminosities classes. The four following figures illustrate some peculiar spectra: Am stars, composite spectra, metal deficient stars and $\mathrm{S}$ and $\mathrm{C}$ stars.

All stars are listed in Tables 1 and 2: Table 1 refers to MK standards and Table 2 to stars with spectral particularities. The tables provide the identifications (HD and HR numbers, name), the spectral classification, the equatorial coordinates for equinox 2000.0, the visual magnitude, the $B-V$ and $R-I$ colour indices, and lastly the number of the figures where the spectrum of the star is illustrated. The data were taken from Hoffleit \& Jaschek (1982), Garcia (1989), Keenan \& McNeil (1976) or from the SIMBAD data base of the Centre de Données de Strasbourg.

\section{a. MK standards}

Nine figures concern the spectral type sequence:

$\begin{array}{llll}\text { F6-K2 V } & \text { F6-K0 III } & \text { G0-G8 Ib } & \text { F5-M2 Ia } \\ \text { K2-M2 V } & \text { K0-M0 III } & \text { G8-K5 Ib } & \\ & \text { M0-M4 III } & \text { K5-M2 Ib } & \end{array}$

Eight figures illustrate the luminosity effects at the following spectral types: F5-6, G0, G2, G5, K0, K2, K5, M2. The F-type spectra in the figures permit to make the link with Part I of the Atlas and are used also for comparison with the Am stars.

In Table 3 we list the stars, ordered by spectral type and luminosity class, so as to permit a quick overview of our coverage. The stars are specified by their HD number.

All MK standards are taken from the list of Garcia (1989) which integrates the different lists given by Morgan and Keenan and their collaborators. The only exception is HD 61064 (F6III) which figures only in the list of Morgan \& Abt (1972).

An identification of the principal lines and features visible on our spectra was made with the help of the solar spectrum (Moore et al. 1966) and is given in Figs. 1 (dwarfs) and 9 (supergiants).

\section{b. Peculiar stars}

- Am stars: Fig. 18.

Even if these stars are of early type, we have included them in this part of the Atlas because very often there do exist confusions between the Am stars and the stars with composite spectra when classified in the $3800-4700 \AA$ region. 
Table 3. MK standard stars ordered by spectral type and luminosity class (identified by $\mathrm{n}^{\circ} \mathrm{HD}$ and illustrated in Figs. 1 to 17$)$

\begin{tabular}{|c|c|c|c|c|}
\hline Spectre & V & III & Ib & Ia \\
\hline F5 & & & & 10494 \\
\hline F6 & 30652 & $\begin{array}{c}61064 \\
160365 \\
\end{array}$ & & \\
\hline G0 & 109358 & 6903 & 26630 & 18391 \\
\hline G1 & 27836 & & & \\
\hline $\mathrm{G} 2$ & $\begin{array}{l}10307 \\
(\mathrm{G} 1.5) \\
\end{array}$ & 161239 & 209750 & \\
\hline G5 & 20630 & 193896 & 206859 & \\
\hline G8 & & & 208606 & \\
\hline K0 & 124752 & 27371 & $\begin{array}{c}221861 \\
\text { (G9) }\end{array}$ & \\
\hline $\mathrm{K} 1$ & & & & $\begin{array}{c}63302 \\
\text { (K1Ia-Iab) }\end{array}$ \\
\hline $\mathrm{K} 2$ & 109011 & 54719 & $\begin{array}{r}210745 \\
\text { (K1.5) } \\
\end{array}$ & \\
\hline K3 & & & 52005 & \\
\hline K5 & 201091 & $\begin{array}{l}17709 \\
\text { (K5.5) }\end{array}$ & 216946 & \\
\hline K7 & 201092 & & 52877 & \\
\hline M0 & $\begin{array}{l}232979 \\
\text { (M0.5) }\end{array}$ & 38944 & & \\
\hline M2 & 1326 & 219734 & 10465 & 206936 \\
\hline M4 & & 76830 & & \\
\hline
\end{tabular}

Their presence in the Atlas shows that even in the near infrared region there exists resemblance between an Am object with a late metallic line type and a composite spectrum of an early A type dwarf and an early G type giant, like in the case of HD 27749, Am, and HD 59604, G2III+A2. The difference appears in the TiI lines $8426 \AA$ and $8435 \AA$ which are very sharp in stars with composite spectra. Also the appearance of the blend $8468 \AA$ permits a separation between the two types of objects.

The three characteristics of the Am stars in our spectral region are:

- the OI $8446 \AA$ line is generally narrow and deep,

- the FeI $8688 \AA$ line is also generally narrow and deep,

- the CaII lines have a very particular aspect, because of their narrowness and the blend with Paschen lines.

In Fig. 18 we show the spectra of two Am stars of very different metallicities: HD 93903 has Paschen lines sim- ilar to those of an A4-5 star, whereas the metallic lines (for instance FeI $8688 \AA$ ) correspond to an early F type; HD 27749 presents Paschen lines similar to those of an A7 star, whereas the metallic lines correspond to a late $\mathrm{F}$ type.

- Stars with composite spectra: Fig. 19.

The composite spectra are produced by the superposition of the spectrum of an early type dwarf of type B or A and that of a late $\mathrm{G}, \mathrm{K}$ and $\mathrm{M}$ giant or supergiant. In the $3800-4800 \AA$ region generally the earlier type spectrum predominates, whereas in the region here reproduced, the spectrum of the late companion is little perturbed by the early type (Ginestet et al. 1994).

Figure 19 presents the spectra of the composites HD 59604-5 and HD $184759-60$ :

In HD 59604 - 5 the hydrogen lines P12 and P14, as well as the OI $8446 \AA$ lines show the presence of a hot object in the system. It should be noticed that the P12 and P14 lines are very weakened because they seem to correspond in intensity to an F2 object, whereas the hotter component is really of type A2. The metallic lines (CaII, FeI, TiI) permit to determine the type of the cool star as being G2III.

In the case of HD $184759-60$, the P12 and P14 lines of the hotter companion are even weaker than in the previous case, they seem to correspond to an F5 star whereas it is really of type A. The metallic lines permit to classify the cool star as being G8III.

- Weak lined stars: Fig. 20.

Figure 20 illustrates the spectra of three metal weak stars: one dwarf HD 6582, $\mu$ Cas, one subdwarf HD 19445 and one giant HD 83632. These spectra are compared to those of two MK standards of type G5V and K2III.

In the spectrum of HD 19445 the CaII lines are very weak and the metallic lines have practically disappeared, except FeI $8688 \AA$ which is faintly visible. The classification attributed in the blue, G5 is difficult to justify a priori on the basis of the near infrared.

HD 83632 appears at a first glance to be a dwarf, but the depth of CaII is larger than in dwarfs and moreover the weakness of FeI $8688 \AA$ does not correspond at all to a dwarf.

- S and C stars: Fig. 21.

Figure 21 shows the spectra of four $\mathrm{S}$ and $\mathrm{C}$ stars: BD $+44^{\circ} 2267$ and HD 112127 may be classified as giant K stars; $\mathrm{ZrO}$ bands are weakly noticeable in their spectrum. HD 187796 ( $\chi$ Cyg) presents the CaII triplet in emission and enhanced absorptions of $\mathrm{TiO}$ whereas HD 92839 is strongly marked by the rotation bands of the red system of CN. The search of the molecular bands was made with the help of Pearse \& Gaydon (1963) for CN, Solf (1978), Huang et al. (1994) for TiO, Davis \& Hammer (1981) for $\mathrm{ZrO}$. 


\section{Classification criteria}

The spectral region is dominated by the CaII triplet lines at $8498 \AA, 8542 \AA$ and $8662 \AA$. The Paschen lines disappear at G0 for class V and III and at G2 for supergiants. Many lines of $\mathrm{FeI}$ and TiI become visible and strengthen with advancing spectral type. We quote specially the $\mathrm{FeI}$ lines at $8621 \AA, 8688 \AA$ and $8514 \AA$ and the TiI lines at $8426 \AA, 8435 \AA$ and $8518 \AA$. Notice also the appearance of molecular bands of $\mathrm{TiO}$ in the coolest stars. All these features can be used for the determination of the spectral type.

Luminosity effects are evident in the line depth. Lines become wider and deeper with higher luminosity (see Figs. 4 to 17). This effect is specially noticeable for the CaII triplet lines. Another good luminosity criterion is the behavior of the blend at $8468 \AA$ (TiI and FeI).

We have provided in Figs. 22 to 27 the curves relating measured equivalent widths to spectral type and luminosity class. These curves are averages based on the measurements from many spectra. The measurements itself were given in Ginestet et al. (1994). The purpose of including these figures is twofold. In first place the figures illustrate very well how the different criteria behave in different spectral types and luminosity classes, so that no lengthy explanation regarding the usefulness of each criterion is needed. In second place the figures illustrate that the equivalent widths can be used to refine the classifications based upon the visual inspection of spectra.

From the Atlas it is clear that it should be possible to obtain from the infrared spectral region classifications which are as precise as those obtained from the blue region. This is a point of real interest for the classification of all red and faint objects.

Acknowledgements. We express our gratitude to Dr. Y. Andrillat from the Université de Montpellier for permitting the use of four spectra for this Atlas. We thank also Prof. P.C. Keenan for useful discussions.

\section{References}

Alloin D., Bica E., 1989, A\&A 217, 57

Andrillat Y., Jaschek C., Jaschek M., 1995, A\&AS 112, 475

Barbieri C., Bonoli C., Bortoletto F., di Serego S. Falomo R., 1981, Mem. Soc. Astron. Ital. 52, 195

Carter D., Visvanathan N., Pickles A.J., 1986, AJ 311, 637

Danks A.C., Dennefeld M., 1994, PASP 106, 382

Davis S.-P., Hammer P.D., 1981, ApJ 250, 805

Diaz A.I., Terlevich E., Terlevich R., 1989, MNRAS 239, 325

Garcia B., 1989, Bull. Inform. CDS 36, 27

Gillet D., Burnage R., Kohler D., et al., 1994, A\&AS 108, 181

Ginestet N., Carquillat J.M., Jaschek M., Jaschek C., 1994, A\&AS 108, 359

Hoffleit D., 1982, "The Bright Star Catalogue", fourth revised edition, Yale Univ. Obs., New Haven, Connecticut

Huang C.C., Friedjung M., Zhou Z.X., 1994, A\&AS 106, 413

Jones J.E., Alloin D.M., Jones B.J.T., 1984, ApJ 283, 457

Keenan P.C., Hynek J.A., 1945, ApJ 101, 265

Keenan P.C., McNeil R., 1976, An Atlas of Stellar Spectra of the Cooler Stars: Types G, K, M, S and C. Colombus. Ohio State University Press

Kirkpatrick J.D., Henry T.J., McCarthy D.W. Jr., 1991, ApJS 77,417

Lemaître G., Kohler D., Lacroix D., Meunier J.P., Vin A., 1990, A\&A 228, 546

Mallik S.V., 1994, A\&AS 103, 279

Merrill P.W., 1934, ApJ 79, 183

Moore Ch.E., Minnaert M.G.J., Houtgast J., 1966, Second Revision of Rowland's Preliminary Table of the Solar Spectrum Wavelengths, N.B.S. Monograph 61

Morgan W.W., Abt H.A., 1972, AJ 77, 35

Parsons S.B., 1964, ApJ 140, 853

Pearse R.W.B., Gaydon A.G., 1963, The Identification of Molecular Spectra, third edition. Chapman \& Hall Ltd., London

Sharpless S., 1956, ApJ 124, 342

Solf J., 1978, A\&AS 34, 409

Torres-Dodgen A.V., Weaver W.B., 1993, PASP 105, 693

Zhou X., 1991, A\&A 248, 367 


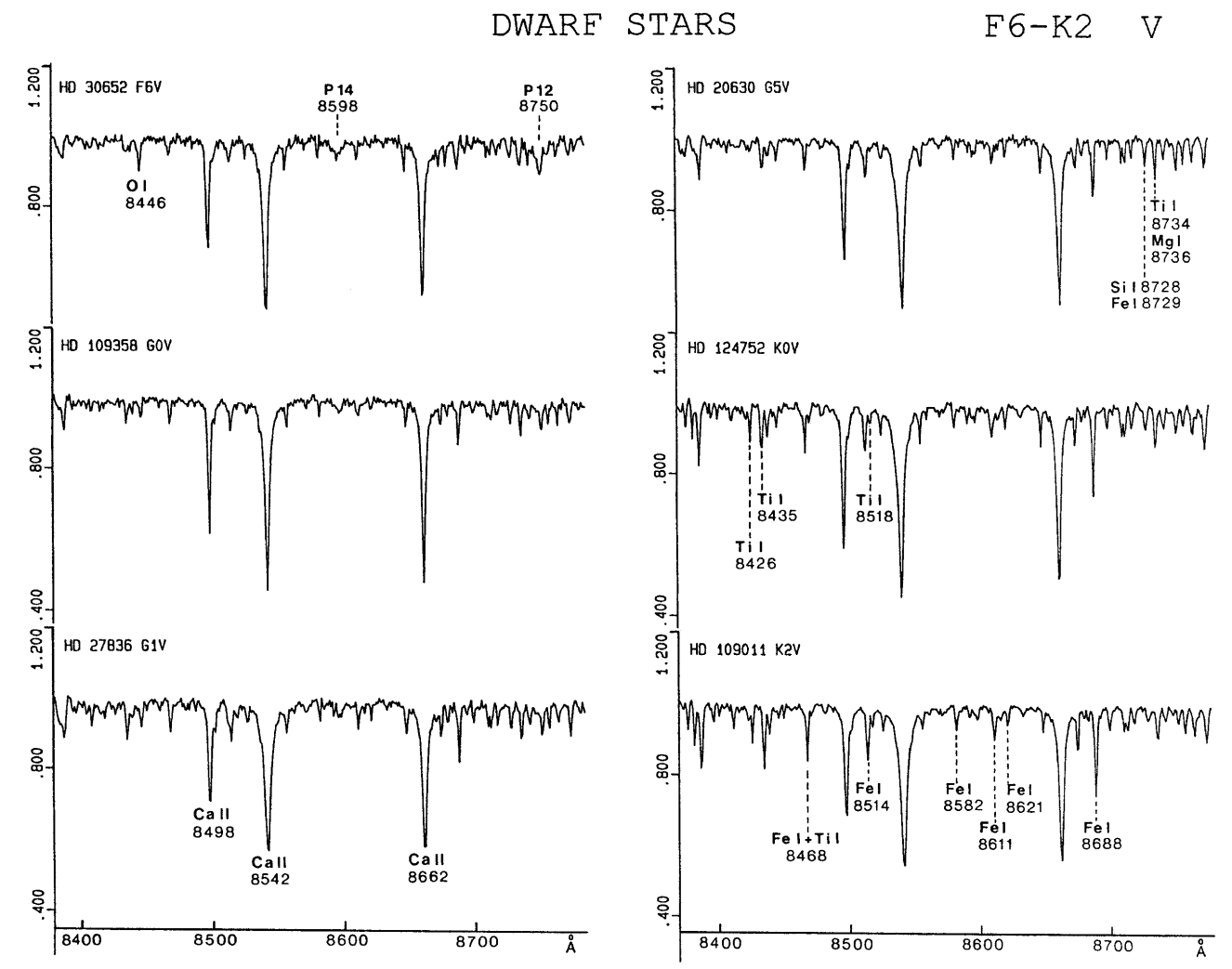

Fig. 1.

DWARE STARS K2-M2 V

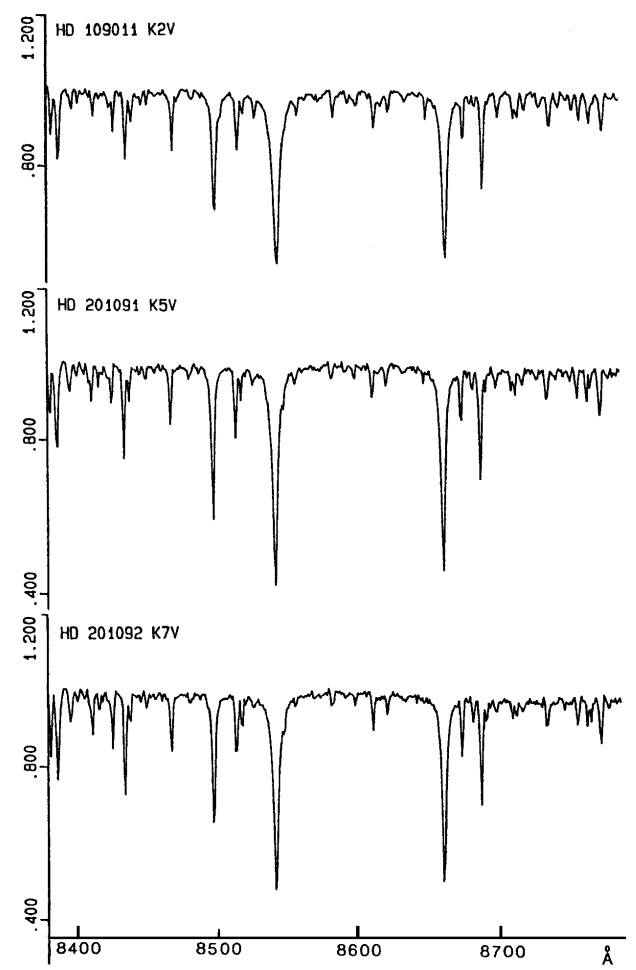

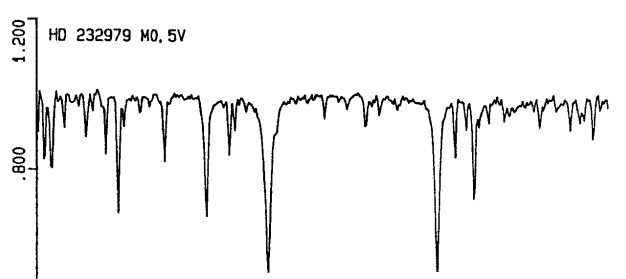

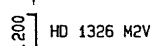

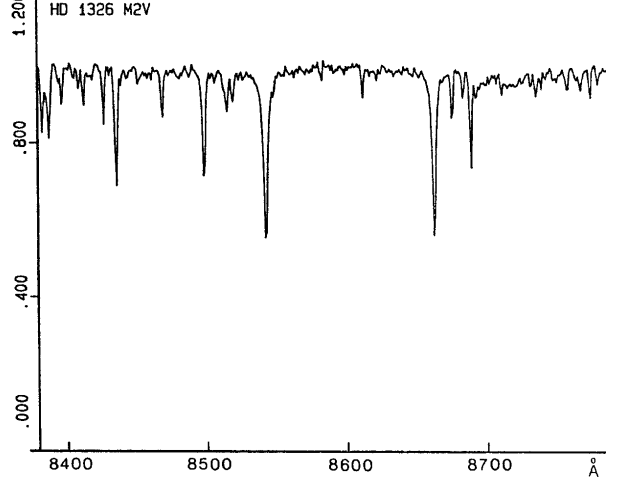

Fig. 2. 
GIANT STARS F6-KO III
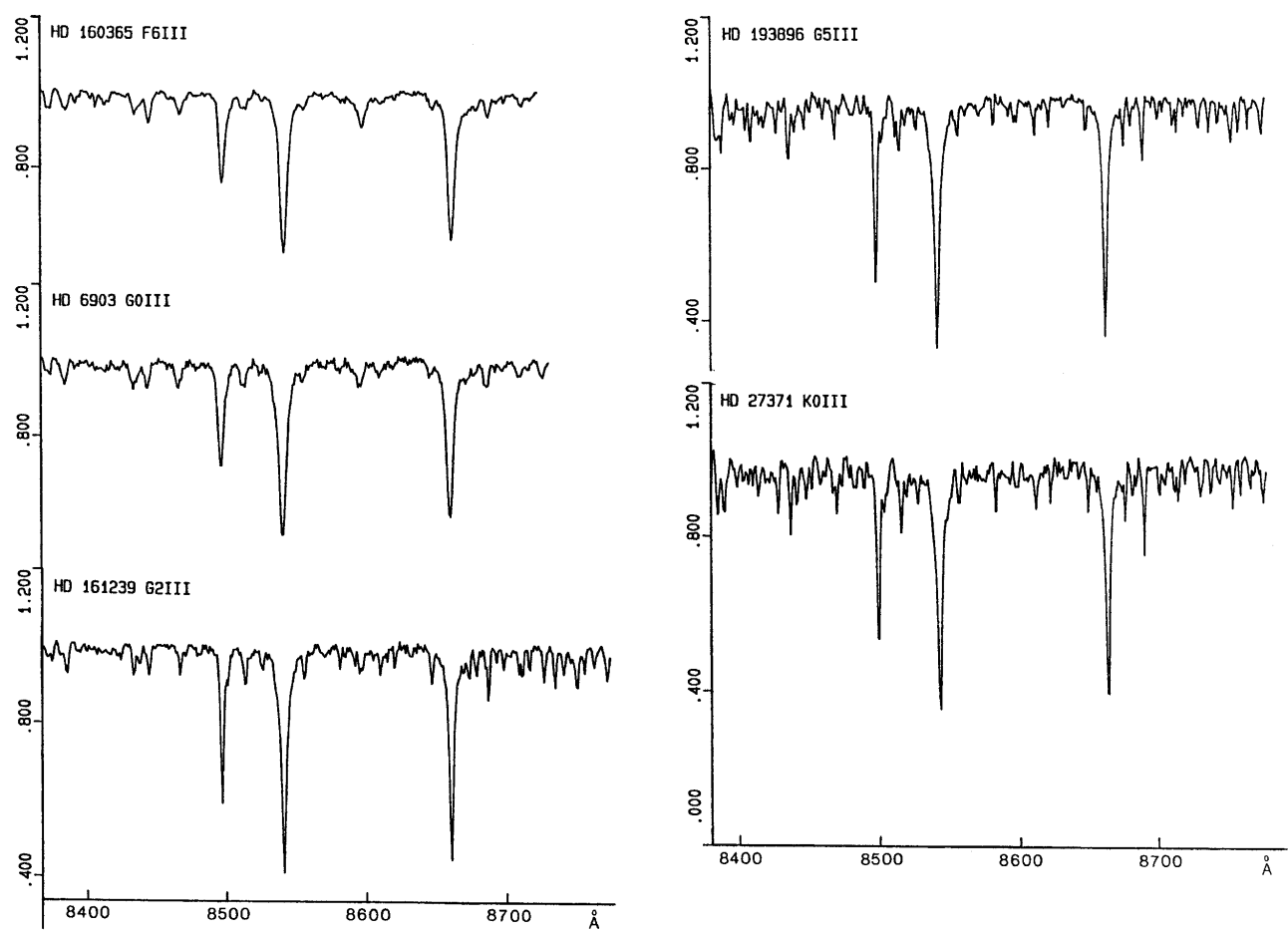

Fig. 3.
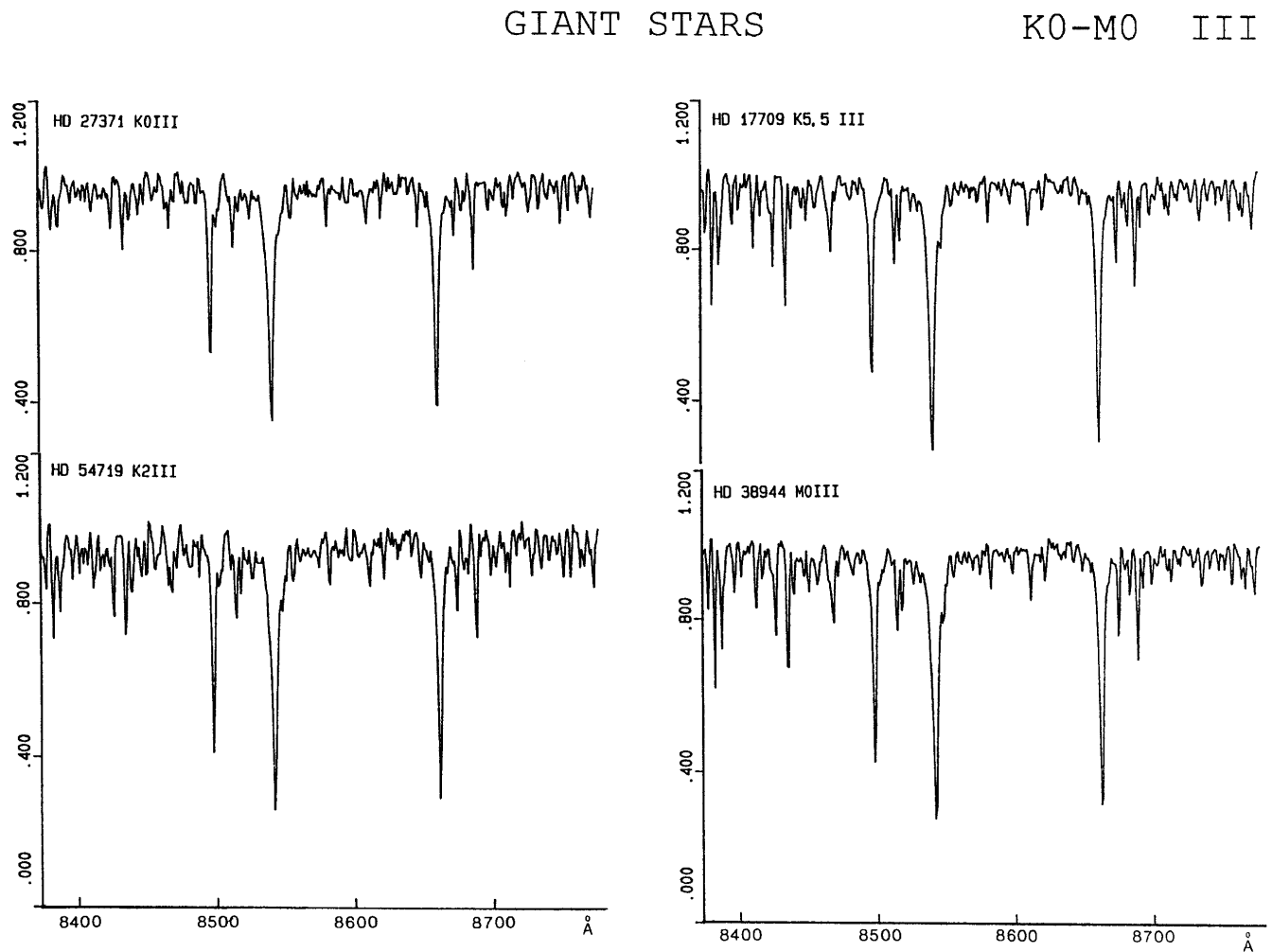

Fig. 4 . 
GIANT STARS MO-M4 III

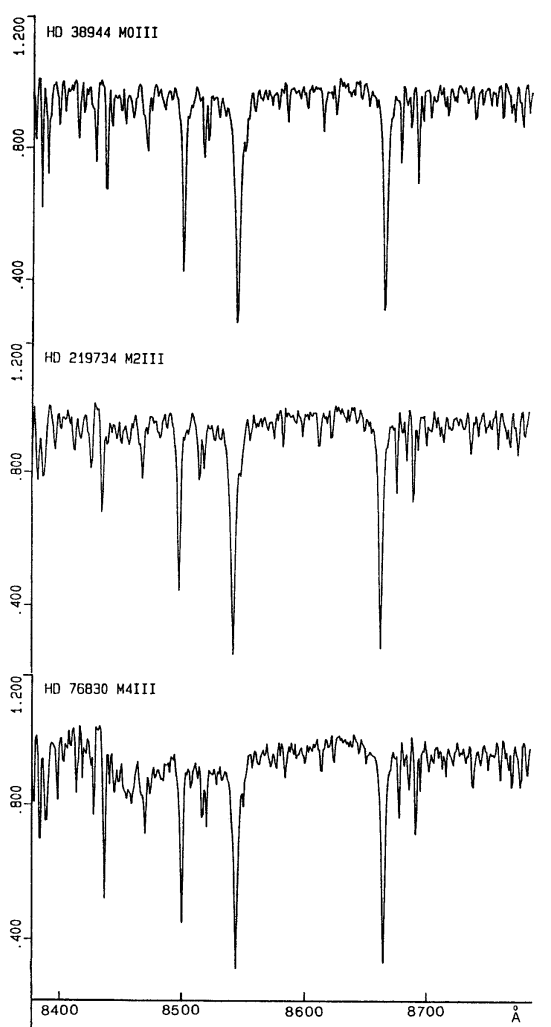

Fig. 5.

SUPERGIANT STARS G0-G8 Ib
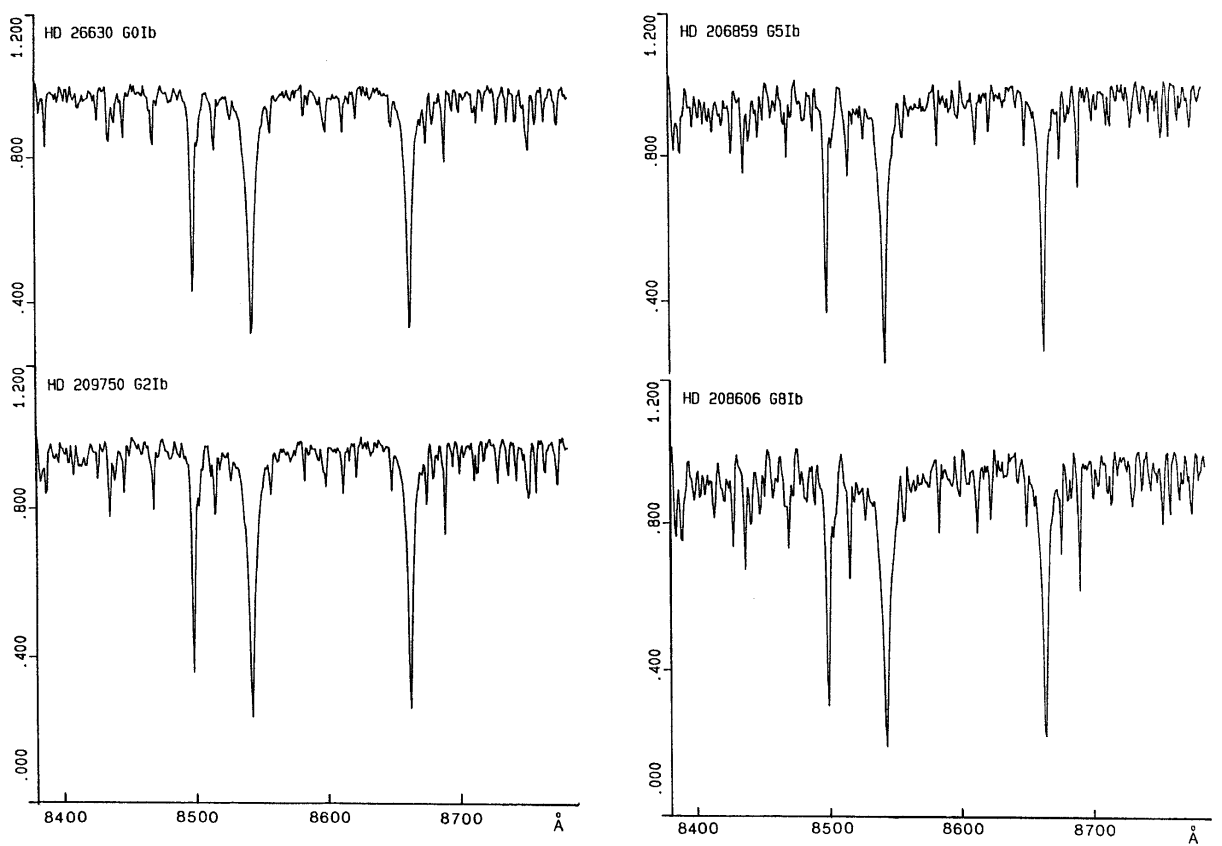

Fig. 6 . 


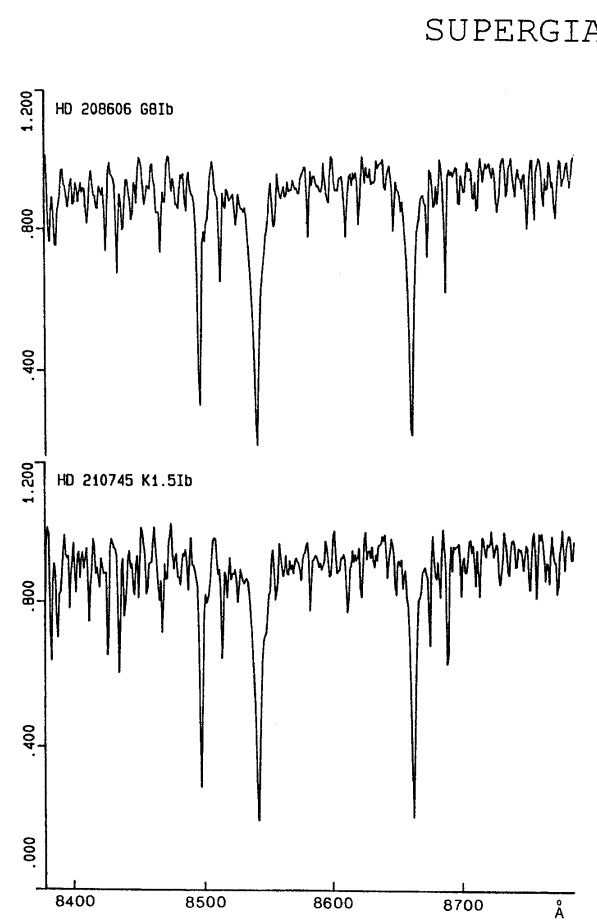

$$
\text { NT STARS G8-K5 Ib }
$$

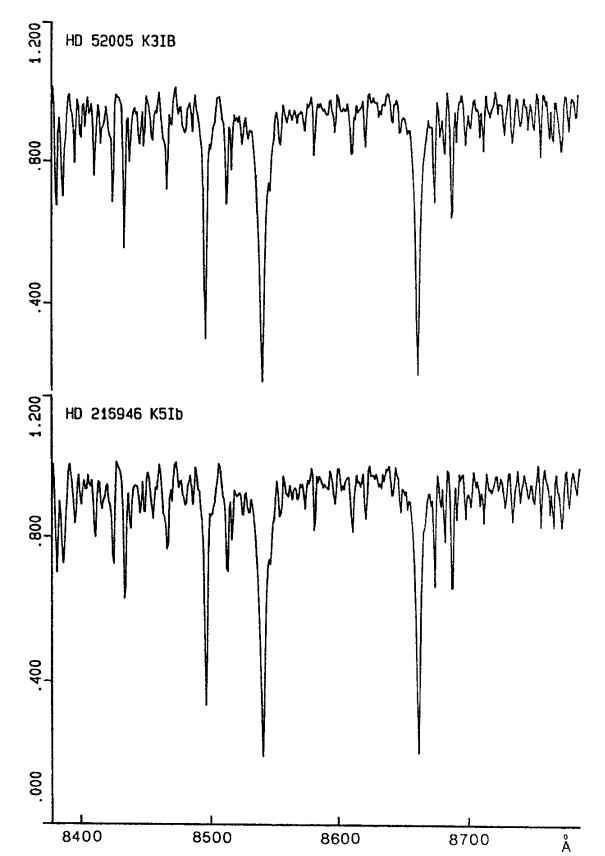

Fig. 7.

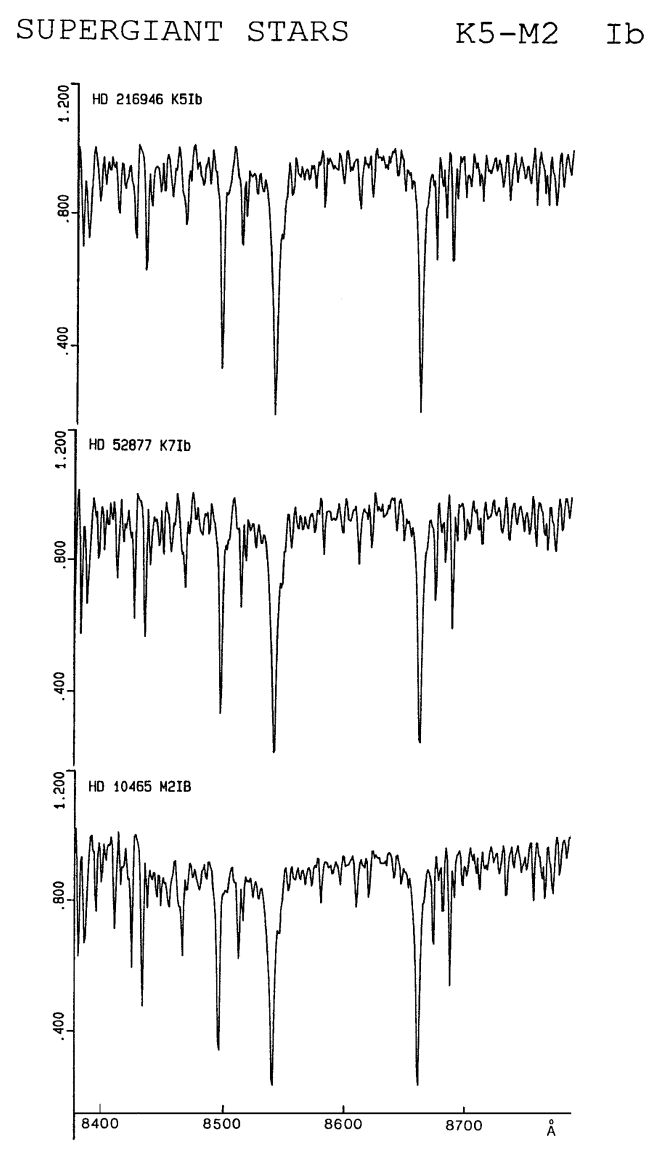

Fig. 8. 


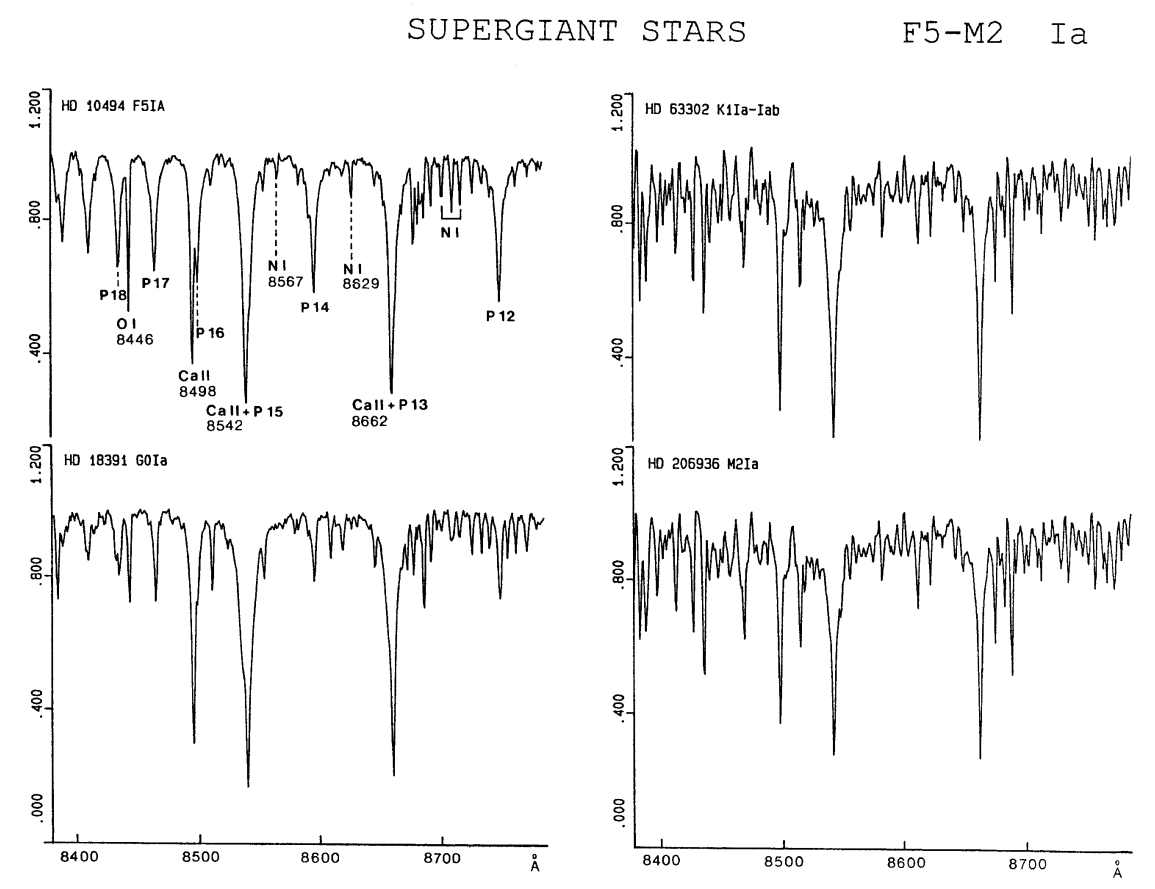

Fig. 9.
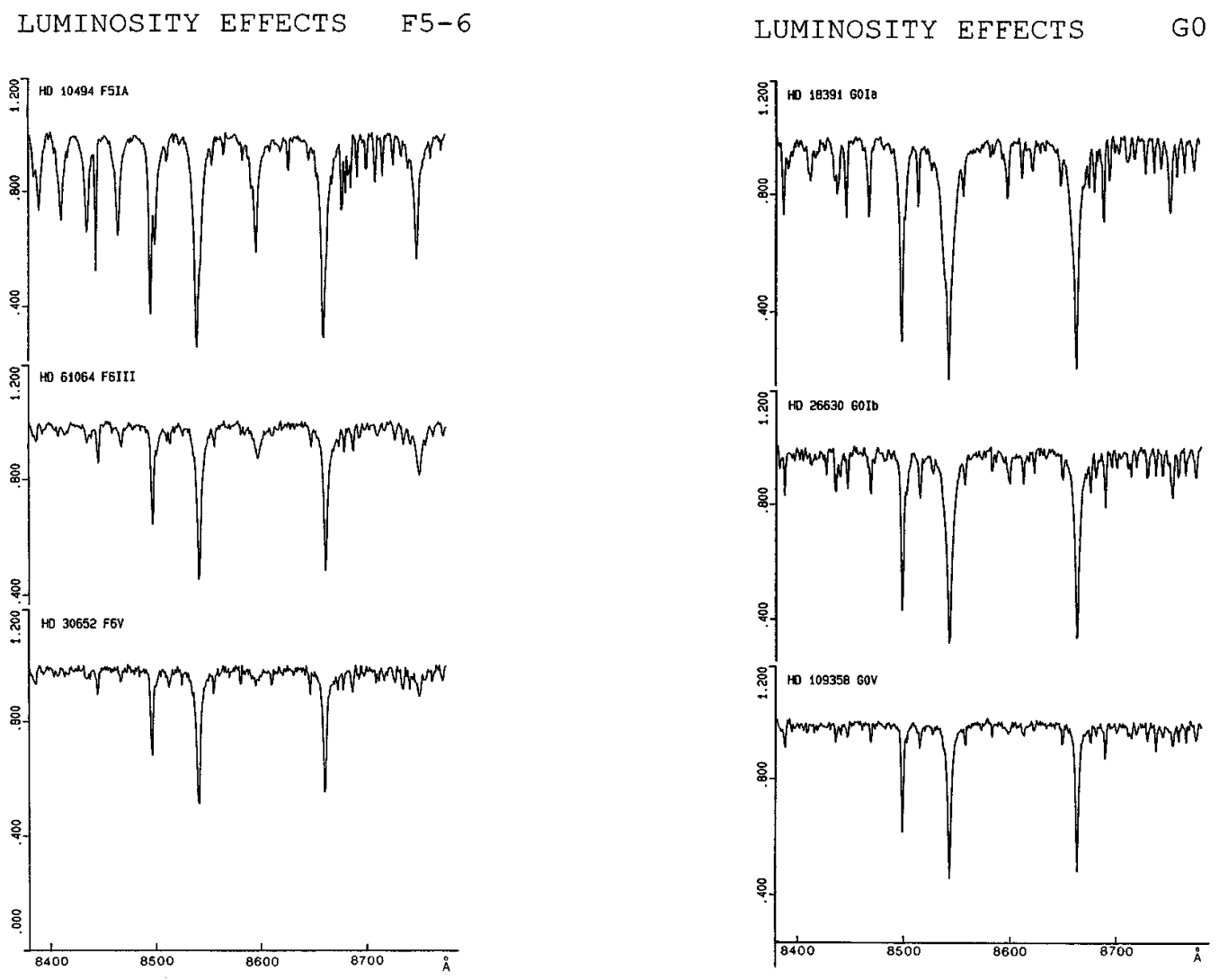

Fig. 10.

Fig. 11. 


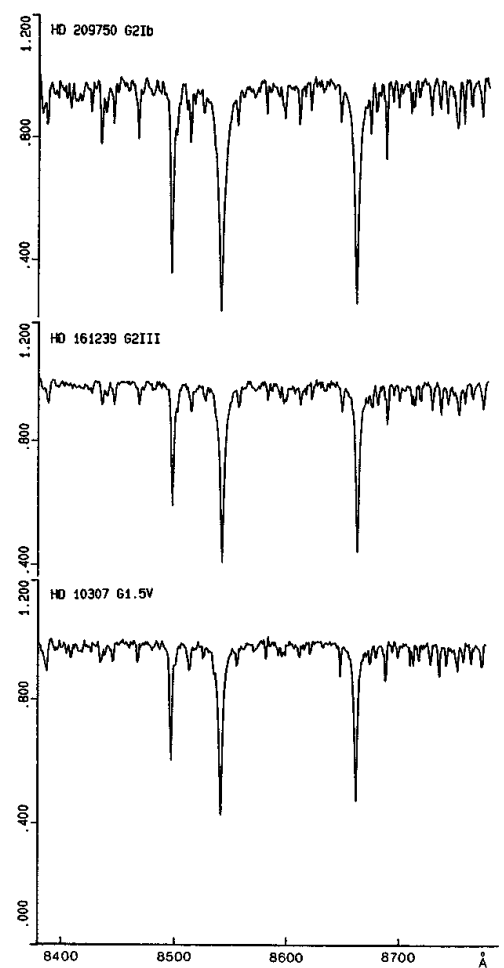

Fig. 12.
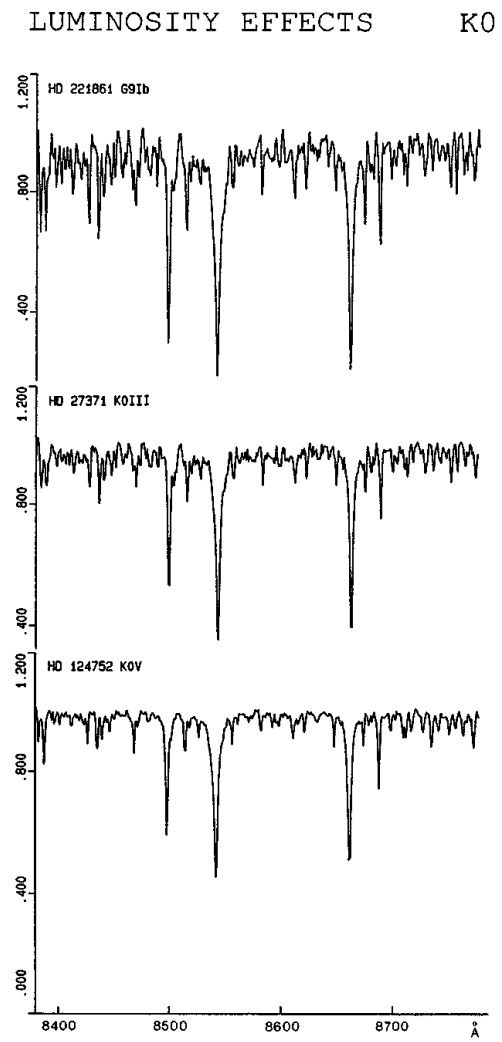

Fig. 14.
LUMINOSITY EFFECTS G5

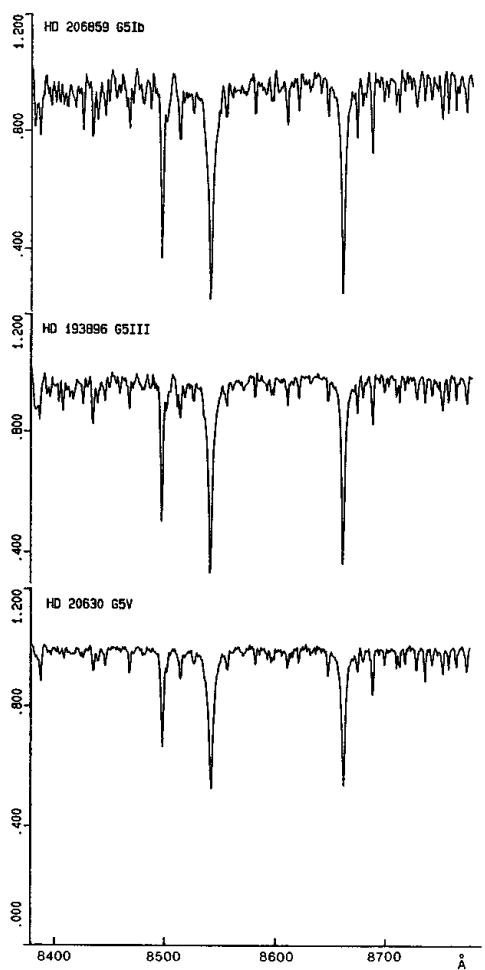

Fig. 13.

LUMINOSITY EFFECTS K2

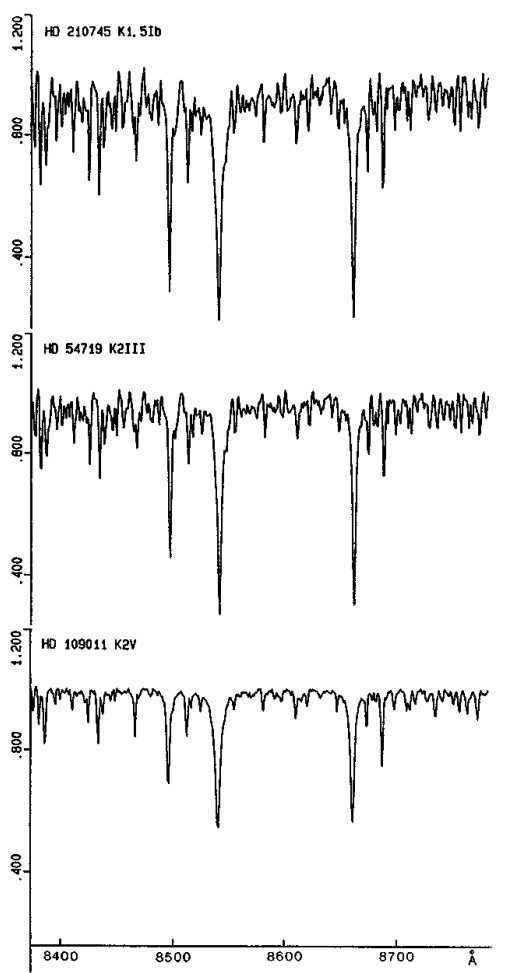

Fig. 15. 


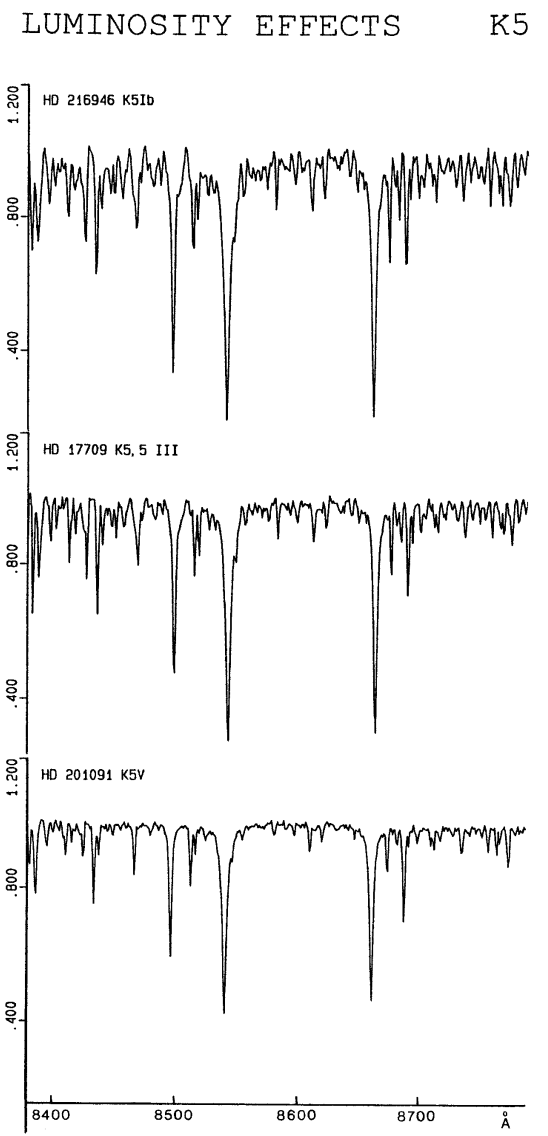

Fig. 16.

LUMINOSITY EEFECTS M2
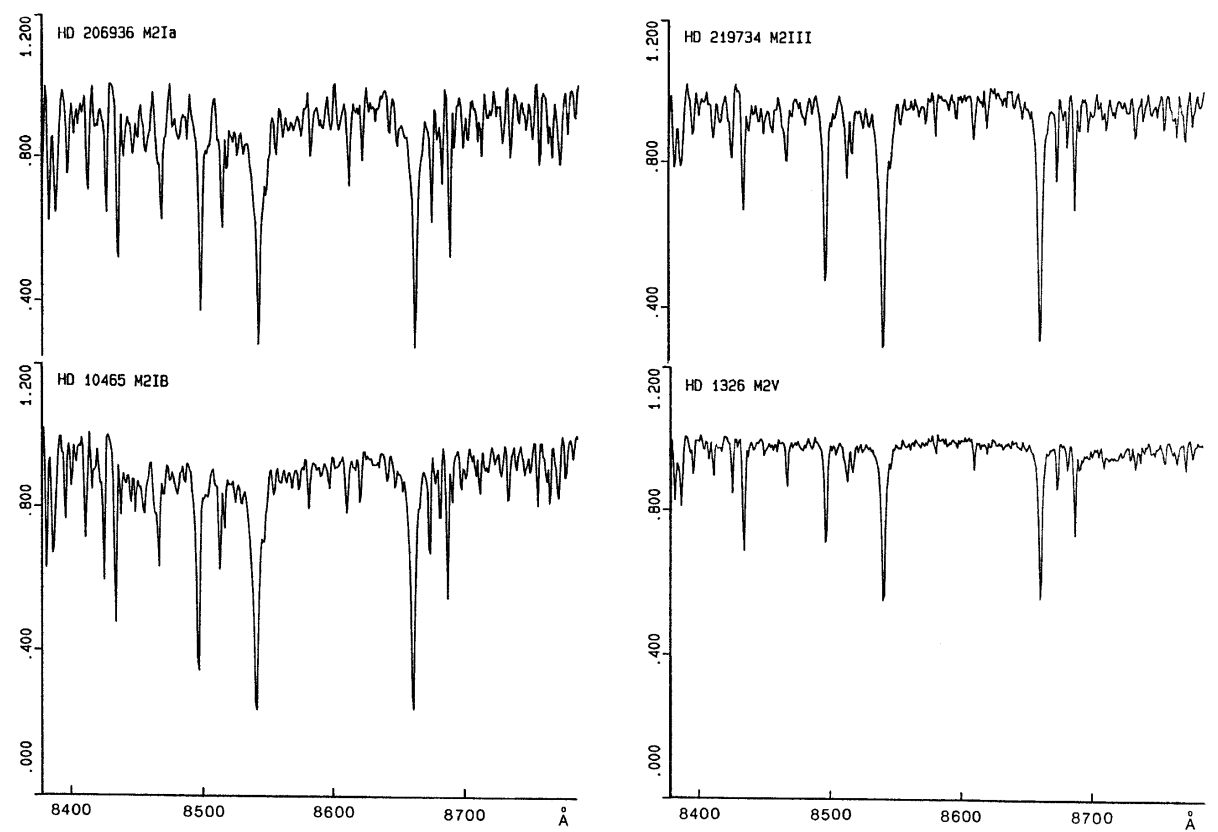

Fig. 17. 
METALLIC LINE STARS Am
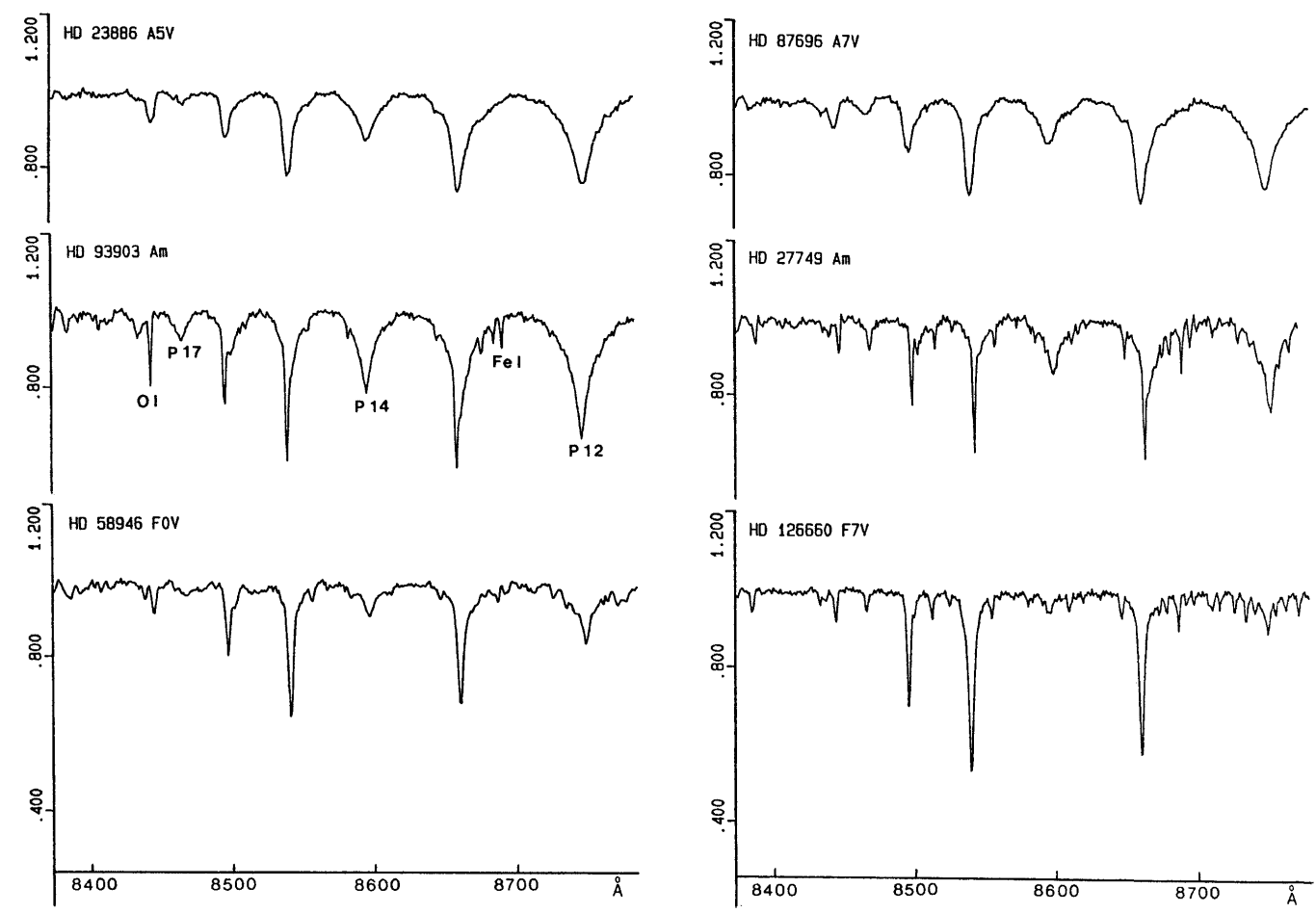

Fig. 18.

\section{STARS WITH COMPOSITE SPECTRA}
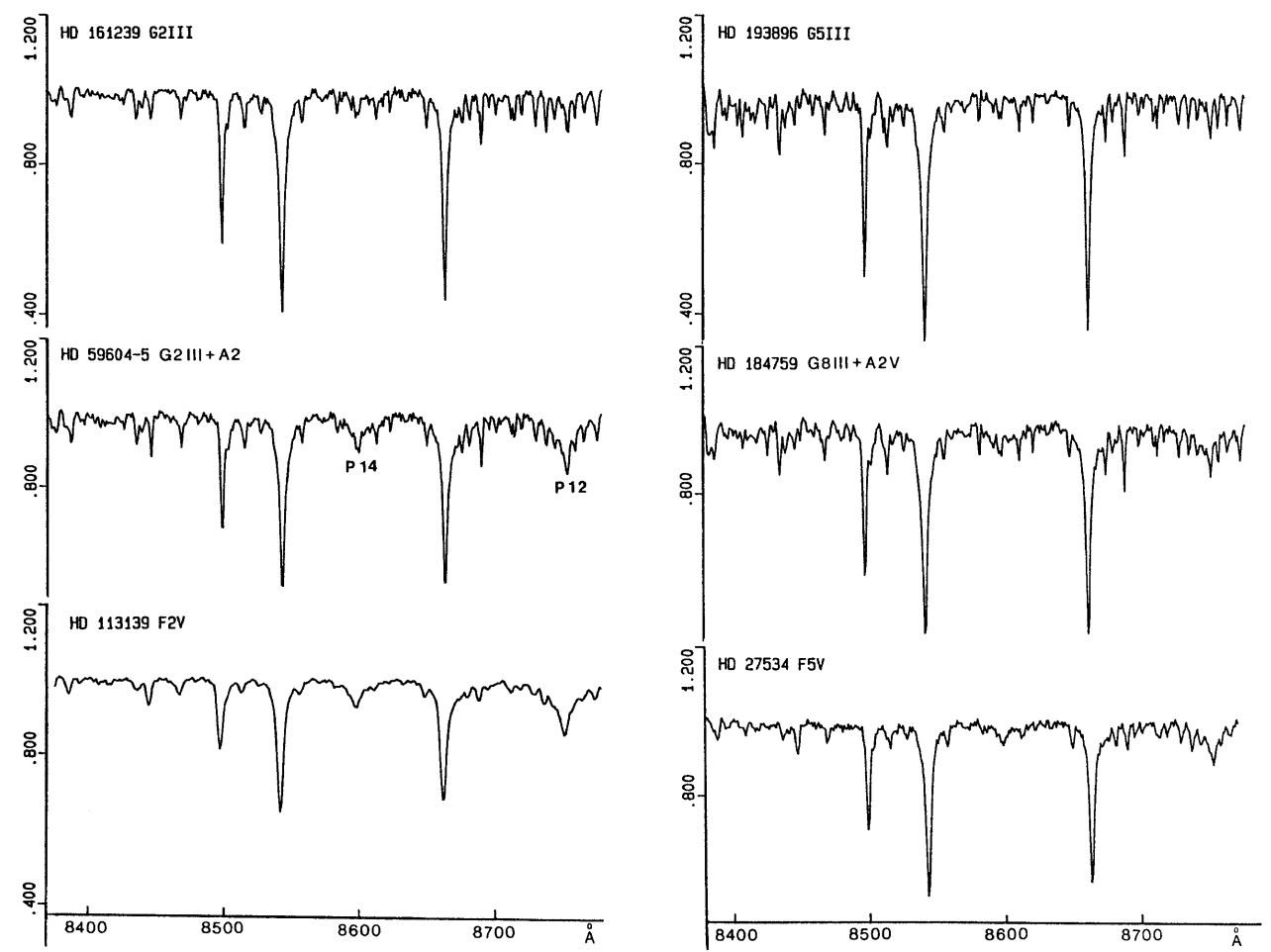

Fig. 19. 
METAL DEFICIENT STARS
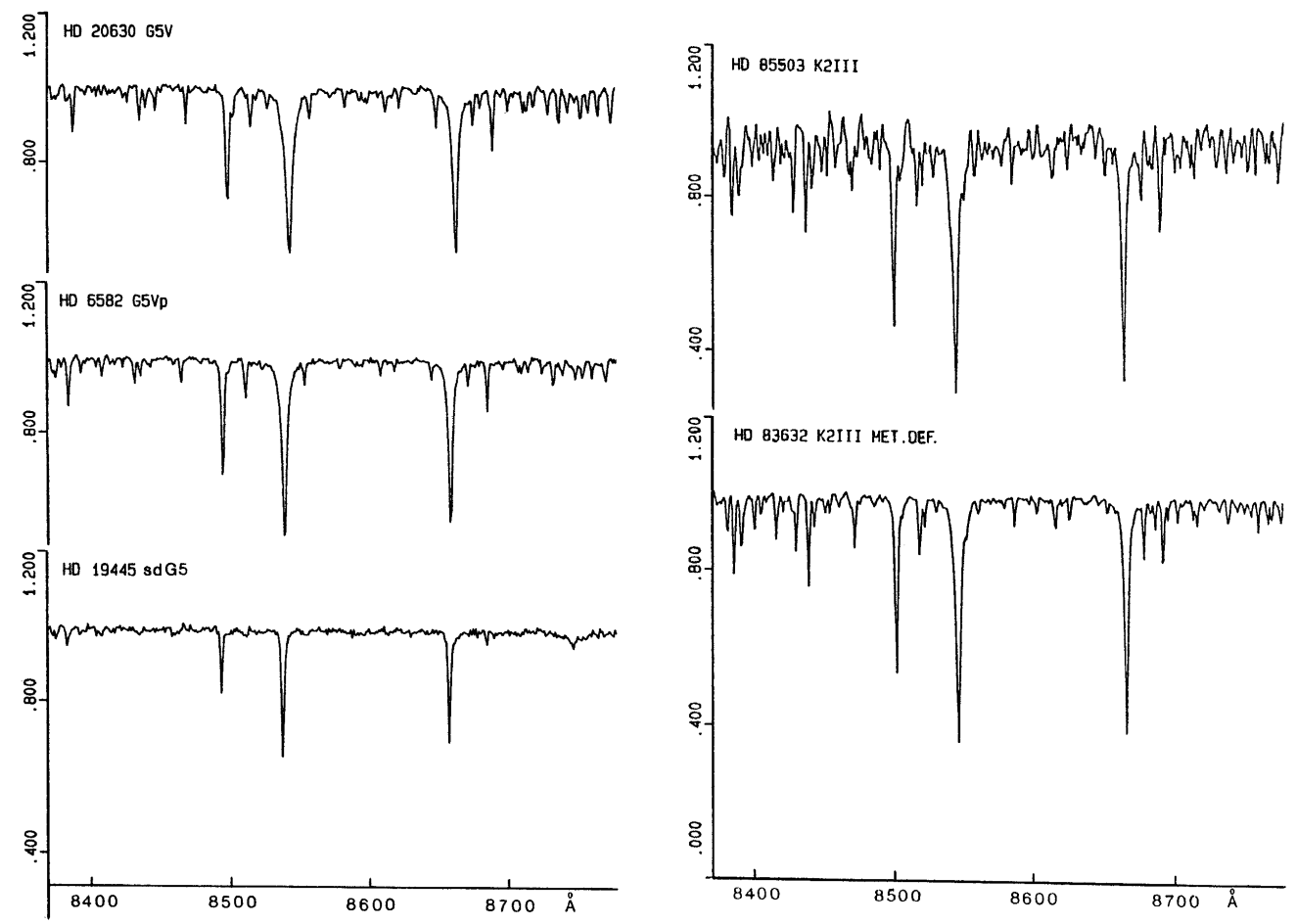

Fig. 20 .

\section{S AND C STARS}
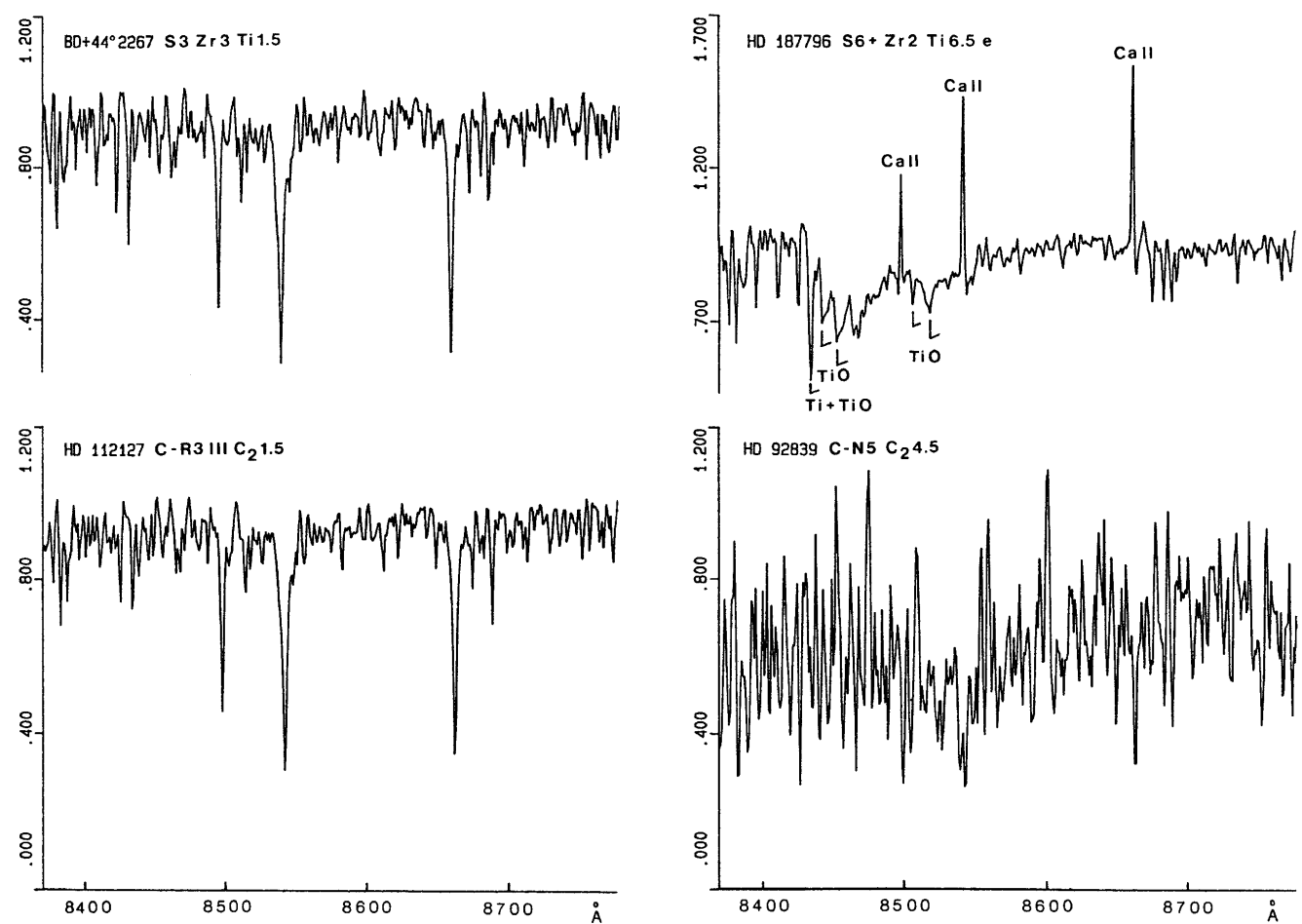

Fig. 21. 


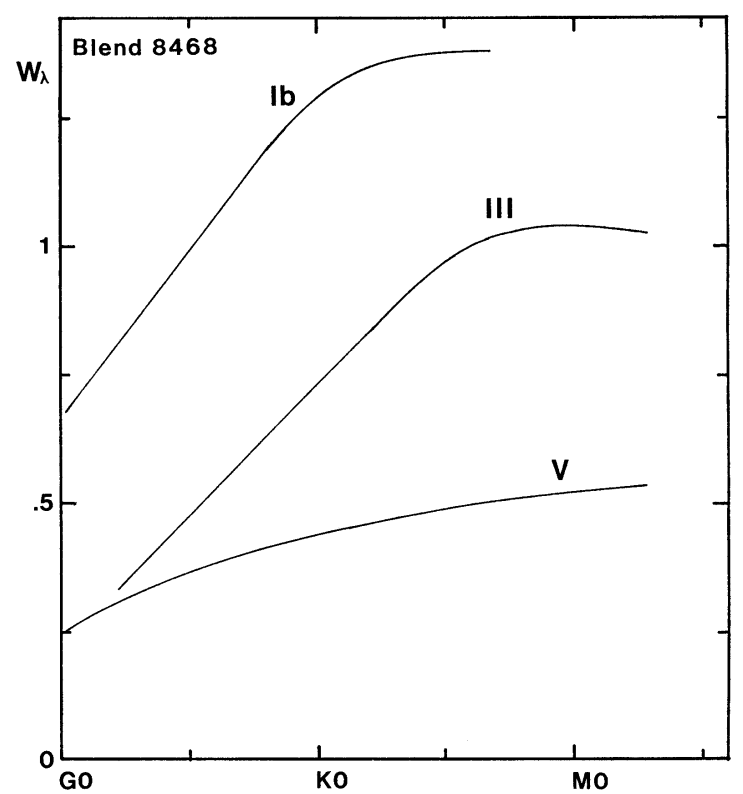

Fig. 22. The equivalent width (in Angström units) of the blend 8468 line as a function of spectral type and luminosity class

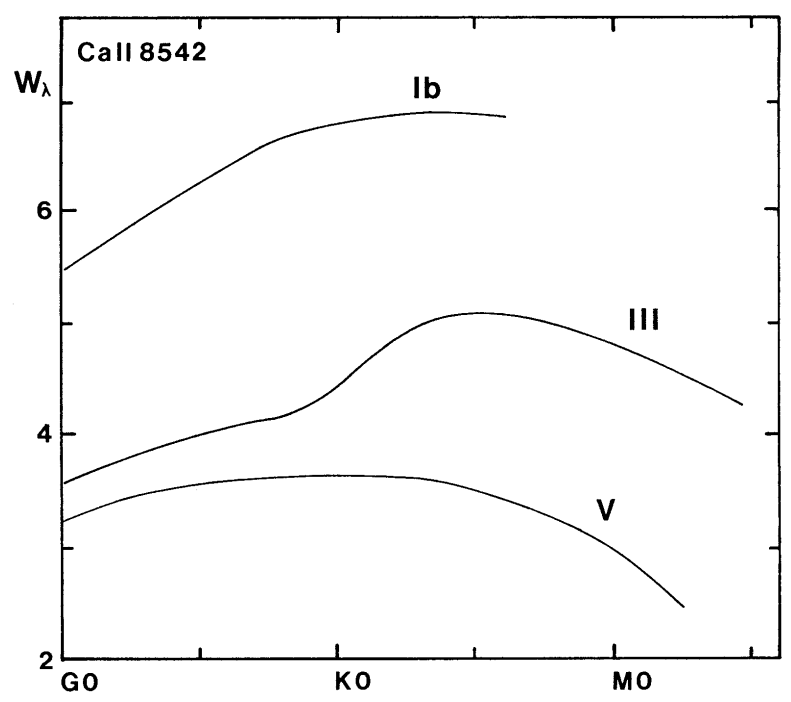

Fig. 23. The equivalent width (in Angström units) of the CaII 8542 line as a function of spectral type and luminosity class

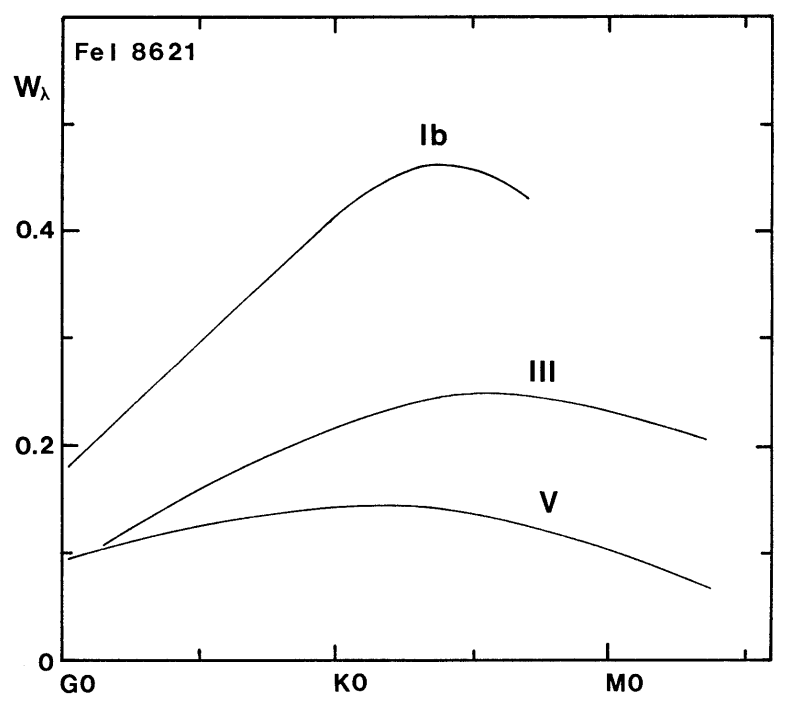

Fig. 24. The equivalent width (in Angström units) of the FeI 8621 line as a function of spectral type and luminosity class

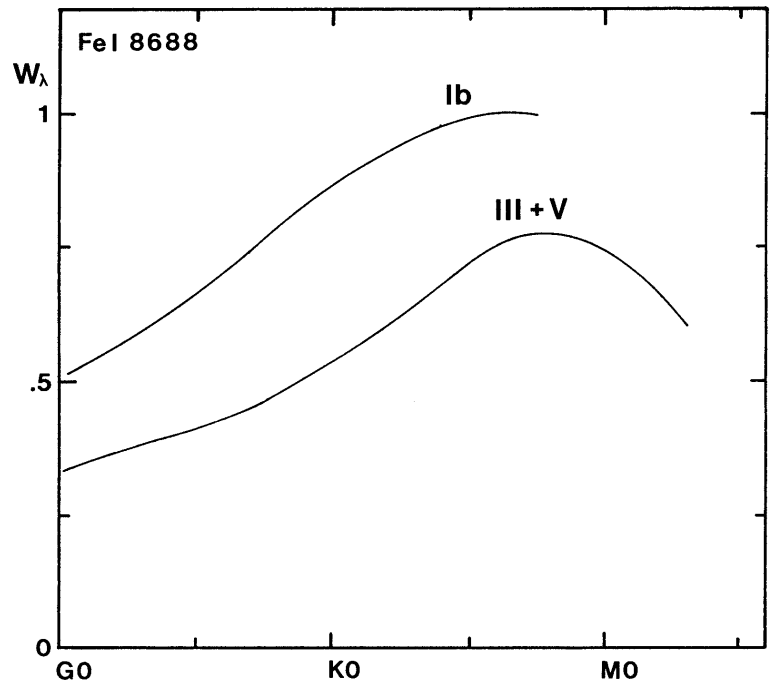

Fig. 25. The equivalent width (in Angström units) of the FeI 8688 line as a function of spectral type and luminosity class 


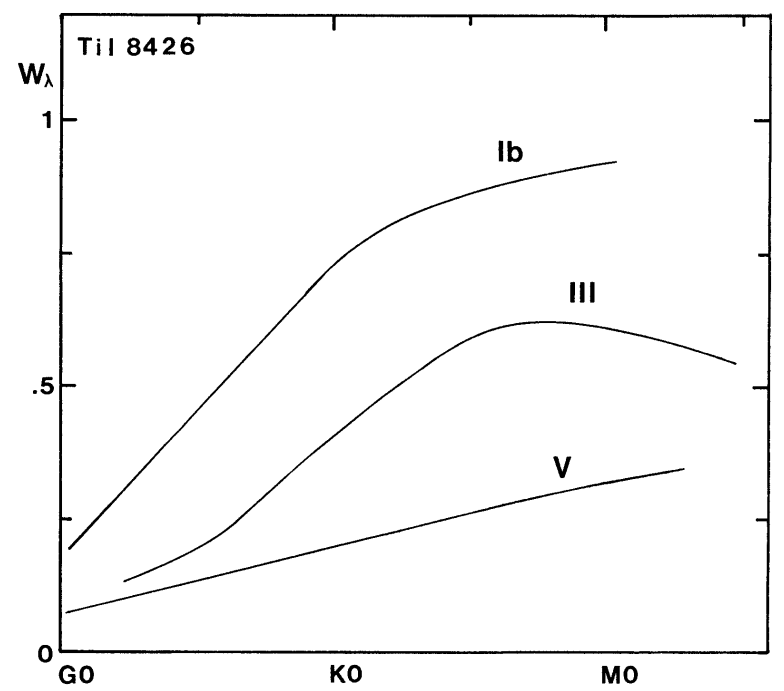

Fig. 26. The equivalent width (in Angström units) of the TiI 8426 line as a function of spectral type and luminosity class

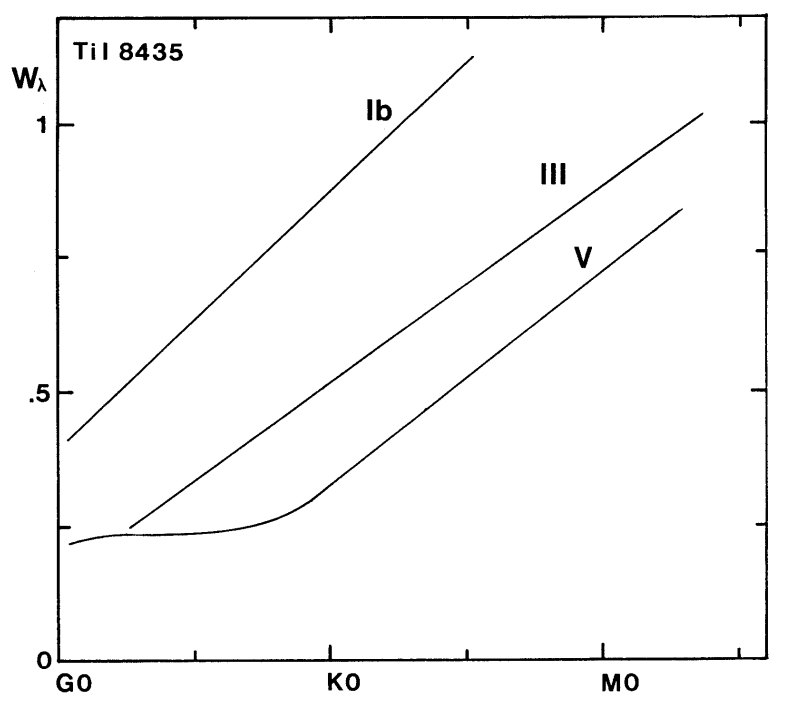

Fig. 27. The equivalent width (in Angström units) of the TiI 8435 line as a function of spectral type and luminosity class 